\title{
Tin halide perovskite films made of highly oriented 2D crystals enable more efficient and stable lead- free perovskite solar cells
}

Meng Li, ${ }^{\dagger}{ }^{\ddagger}, \S$ Wei-Wei Zuo, ${ }^{\ddagger}, ~ "$ Ying-Guo Yang, ${ }^{\perp}$ M. H. Aldamasy, ${ }^{\ddagger} \#$, Qiong Wang, ${ }^{\ddagger}$ Silver Hamill Turren Cruz, ${ }^{\ddagger}$ Shang-Lei Feng, ${ }^{\perp}$ Michael Saliba, ${ }^{\|, ~} \nabla$ Zhao-Kui Wang, ${ }^{*, \dagger}$ and Antonio Abate $^{*, 亠, \emptyset}$

$\dagger$ Jiangsu Key Laboratory for Carbon-Based Functional Materials \& Devices, Institute of Functional Nano \& Soft Materials (FUNSOM), Soochow University, Suzhou 215123, China

Helmholtz-Zentrum Berlin für Materialien und Energie, Kekuléstraße 5, 12489 Berlin, Germany

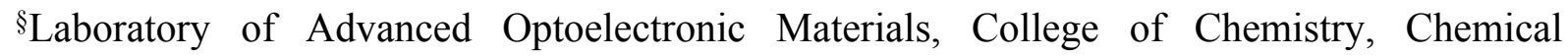
Engineering and Materials Science, Soochow University, Suzhou 215123, China

\|Institute of Materials Science, Technische Universität Darmstadt, Alarich-Weiss-Straße 2, 64287 Darmstadt, Germany

${ }^{\perp}$ Shanghai Synchrotron Radiation Facility (SSRF), Shanghai Advanced Research Institute, Chinese Academy of Sciences, 239 Zhangheng Road, Shanghai 201204, P. R. China \#Egyptian Petroleum Research Institute, Nasr City, P.O. 11727 Cairo, Egypt

${ }^{\nabla}$ Helmholtz Young Investigator Group FRONTRUNNER Forschungszentrum Jülich GmbH, Institut für Energie- und Klimaforschung (IEK-5), 52425 Jülich, Germany

"Department of Chemical, Materials and Production Engineering, University of Naples Federico II, Piazzale Tecchio 80, 80125 Fuorigrotta, Naples, Italy

*Corresponding author: zkwang@suda.edu.cn, antonio.abate@helmholtz-berlin.de 


\section{EXPERIMENTAL SECTION}

\section{Materials Preparation.}

Synthesis of non-stoichiometric $\mathrm{NiO}_{x}$ nanoparticles: 1 mol Nickel nitrate hexahydrate (Aladdin) was dispersed in $200 \mathrm{~mL}$ of deionised water to obtain a dark green solution. And then $\mathrm{NaOH}$ solution $\left(10 \mathrm{~mol} \mathrm{~L}^{-1}\right)$ was added to adjust the $\mathrm{pH}$ of the solution to 10 . After stirring for $5 \mathrm{~min}$, the precipitate was thoroughly washed twice with deionised water and dried at $80{ }^{\circ} \mathrm{C}$ for $6 \mathrm{~h}$. The resulting green powder was then calcined at $270{ }^{\circ} \mathrm{C}$ for 2 hours. After that we can get a dark black powder. Finally, the obtained nanoparticles are dispersed in deionised water to 10 $\mathrm{mg} \mathrm{ml}^{-1} \cdot 1,2$

Clevios VP AI 4083 PEDOT:PSS water dispersion was acquired from Heraeus (Germany). phenyl ethyl ammonium chloride (PEACl) and Formamidinium iodide (FAI) were purchased from TCI EUROPE N.V. Tin (II) iodide ( $\left.\mathrm{SnI}_{2}, 99.999 \%\right)$, tin (II) fluoride $\left(\mathrm{SnF}_{2}, 99 \%\right)$, Buckminsterfullerene (C60, 99.5\%), bathocuproine (BCP, 99.9\%), dimethyl sulfoxide (DMSO), and chlorobenzene (CB) were purchased from Sigma Aldrich. All the chemicals were used as received without further purification.

\section{Device Fabrication.}

The FTO-coated glass substrate $\left(15 \Omega \mathrm{sq}^{-1}\right)$ was thoroughly washed with detergent, acetone, ethanol, deionised water, and isopropanol, respectively, each for 15 minutes in an ultrasonic bath. A PEDOT:PSS layer was spin-coated onto the FTO substrates at $4000 \mathrm{rpm}$ for $40 \mathrm{~s}$ and dried at $100{ }^{\circ} \mathrm{C}$ for $30 \mathrm{~min}$. Or a $\mathrm{NiO}_{\mathrm{x}}$ layer was spin-coated onto the FTO substrates at 5000 rpm for $40 \mathrm{~s}$ and dried at $150{ }^{\circ} \mathrm{C}$ for $30 \mathrm{~min}$. The coated samples were then transferred to a nitrogen-filled glove-box. SnI2 (409 mg), FAI (189 mg), and SnF2 (17.3 mg) were dissolved in the solvent of $1 \mathrm{~mL}$ DMSO, then add PEACl solution into perovskite solution without or with different doping concentration. The control $\mathrm{FASnI}_{3}$ or $\mathrm{FASnI}_{3}: \mathrm{PEACl}$ (with different doping concentration) films were spin-coated from a precursor solution at $4000 \mathrm{rpm}$ for $70 \mathrm{~s}$. chlorobenzene was used as the antisolvent during the spin-coating process. The $\mathrm{FASnI}_{3}$ or 
$\mathrm{FASnI}_{3}: \mathrm{PEACl}$ films were then annealed at different temperature $\left(60^{\circ} \mathrm{C}, 70^{\circ} \mathrm{C}, 80^{\circ} \mathrm{C}, 90^{\circ} \mathrm{C}\right.$, $\left.100^{\circ} \mathrm{C}\right)$. Then, $\mathrm{C}_{60}(23 \mathrm{~nm}) / \mathrm{BCP}(8 \mathrm{~nm}) / \mathrm{Ag}(100 \mathrm{~nm})$ were sequentially thermally evaporated onto the perovskite layer. The effective area of the perovskite solar cells is $0.18 \mathrm{~cm}^{2}$.

SEM images. The field-emission scanning electron microscope (SEM) images were obtained from a Quanta 200 FEG (FESEM, FEI Co.).

XRD and GIXRD measurement. X-ray diffraction (XRD) measurement was conducted using PANalytical (Empyrean) equipment. The grazing incidence X-ray diffraction (GIXRD) measurements were performed at the BL14B1 beamline of the Shanghai Synchrotron Radiation Facility (SSRF) using X-ray with a wavelength of $0.6887 \AA$. Two-dimensional (2D) GIXRD patterns were acquired by a MarCCD mounted vertically at a distance $\sim 397$ $\mathrm{mm}$ from the sample with a grazing incidence angle of $0.2^{\circ}$, and an exposure time of 60 sec. The 2D GIXRD patterns were analysed using the FIT2D software and displayed in scattering vector $q$ coordinates.

X-ray photoelectron spectra (XPS) were obtained from a Thermo K-ALPHA Surface Analysis for perovskite films.

Solar cell characterisation. Current density-voltage characteristics of perovskite solar cells under 1 sun illumination were performed using a programmable Keithley 2400 source meter under AM 1.5G solar irradiation at $100 \mathrm{~mW} \mathrm{~cm}^{-2}$ (Newport, Class AAA solar simulator, $94023 \mathrm{~A}-\mathrm{U}) . J-V$ curves were measured at a scan rate of $0.1 \mathrm{~V} \mathrm{~s}^{-1}$, and the time interval was 50 ms. The incident-photon-to-current efficiency (IPCE) measurement was performed using a system combining a xenon lamp, a monochromator, a chopper and a lock-in amplifier with a calibrated silicon photodetector. 


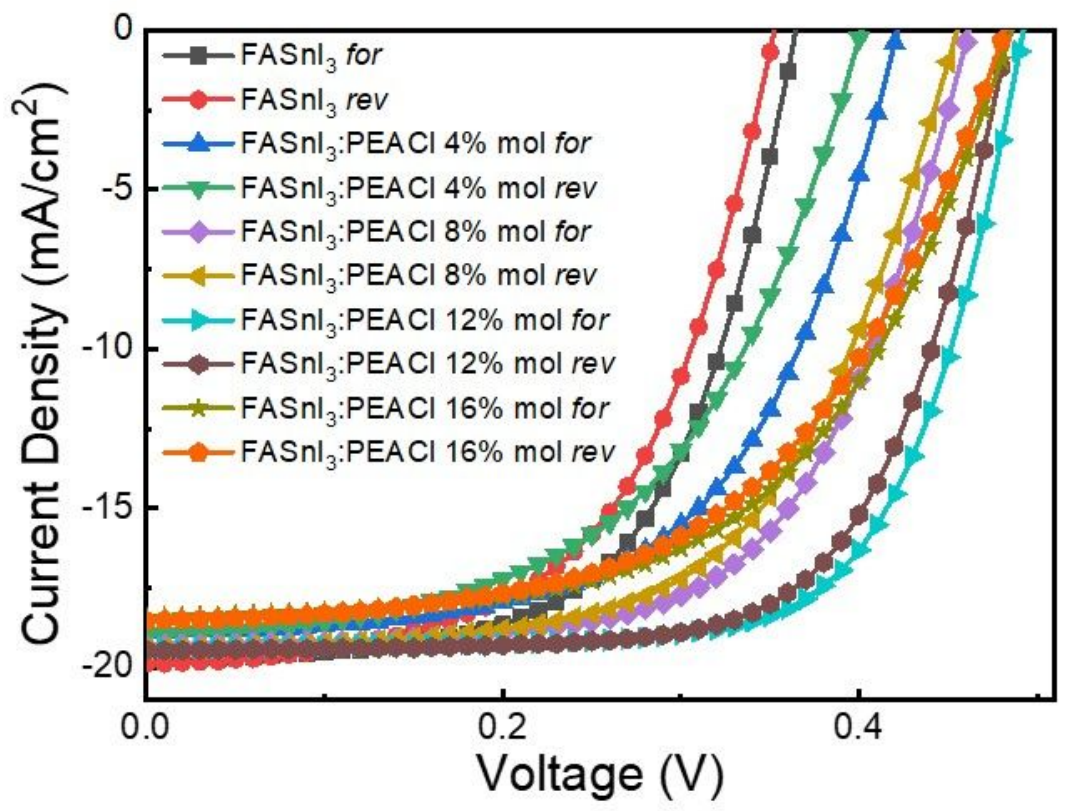

Figure S1. Forward and reverse sweep $J-V$ curves of $\mathrm{FASnI}_{3}$ perovskite solar cells without and with $\mathrm{PEACl}$ at different concentrations. 


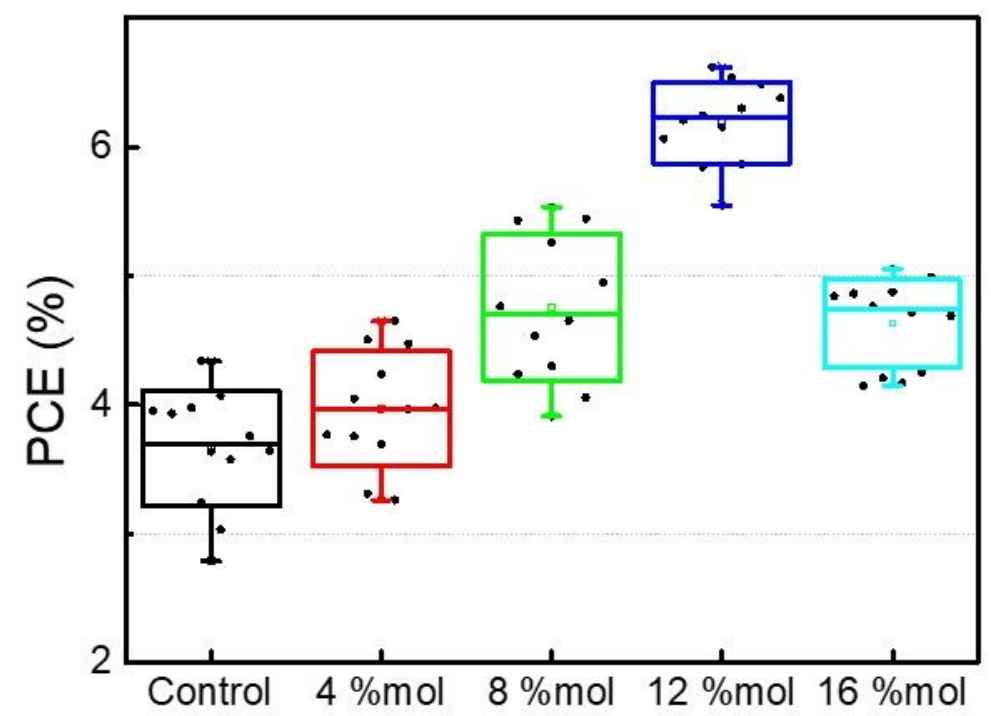

Figure S2. Box plots of photovoltaic parameters for the $\mathrm{FASnI}_{3}$ perovskite solar cells without and with $\mathrm{PEACl}$ at different concentrations. 


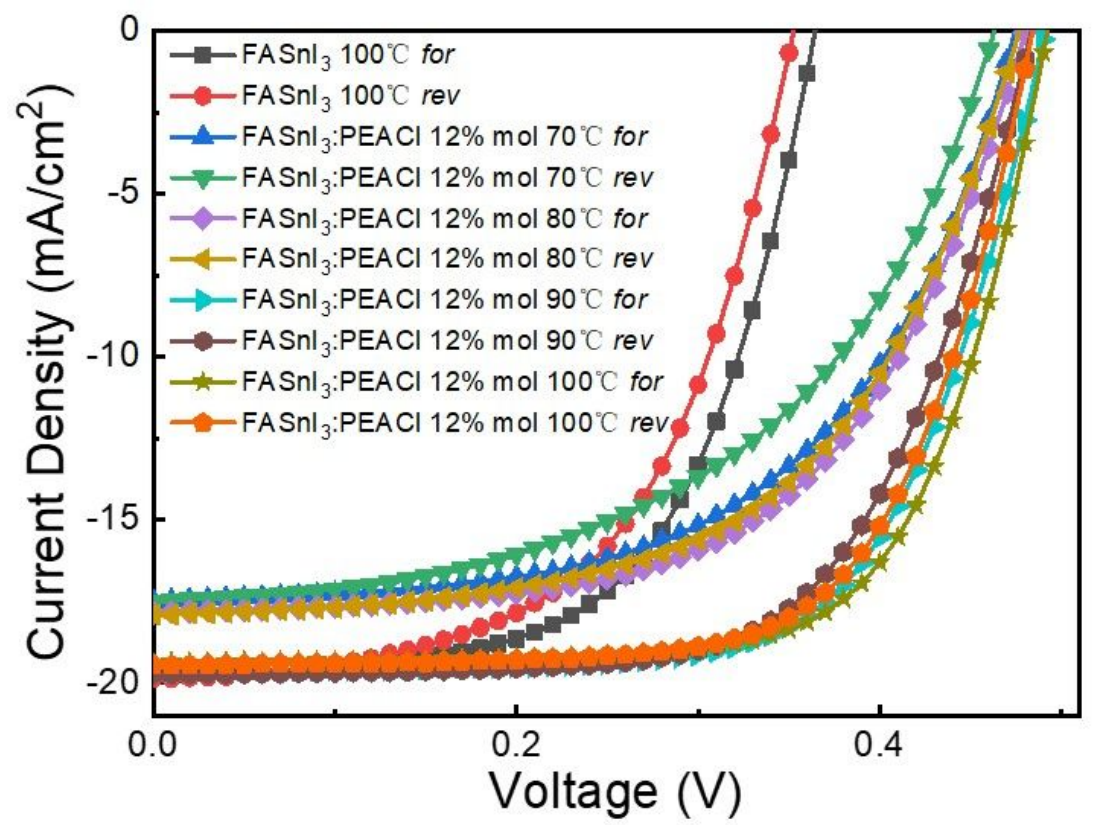

Figure S3. Forward and reverse sweep $J-V$ curves of $\mathrm{FASnI}_{3}: \mathrm{PEACl}$ perovskite solar cells with different annealing processes. 

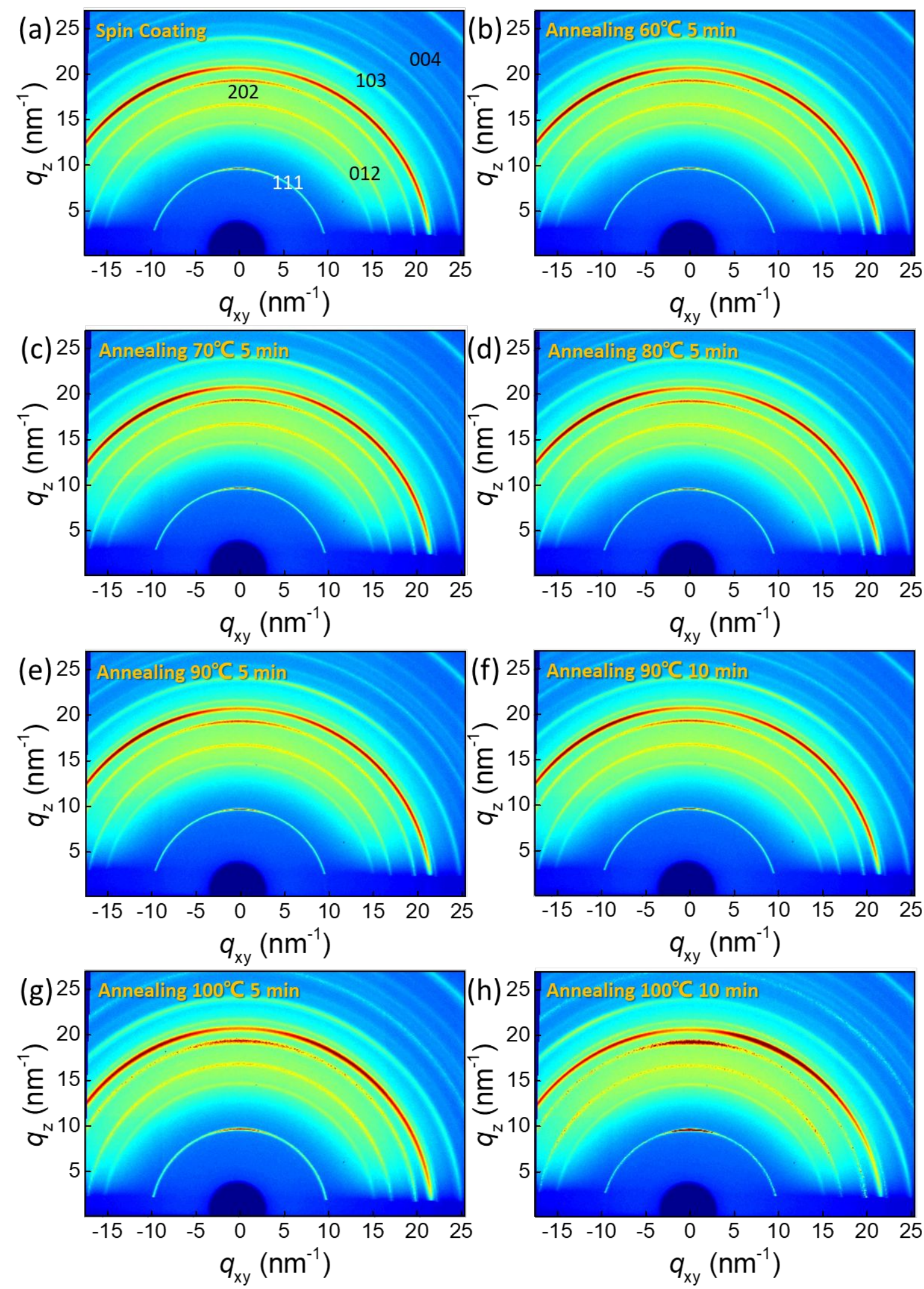

Figure S4. 2D GIXRD patterns of $\mathrm{FASnI}_{3}$ films (a) without annealing and (b-h) annealing at different temperatures. 

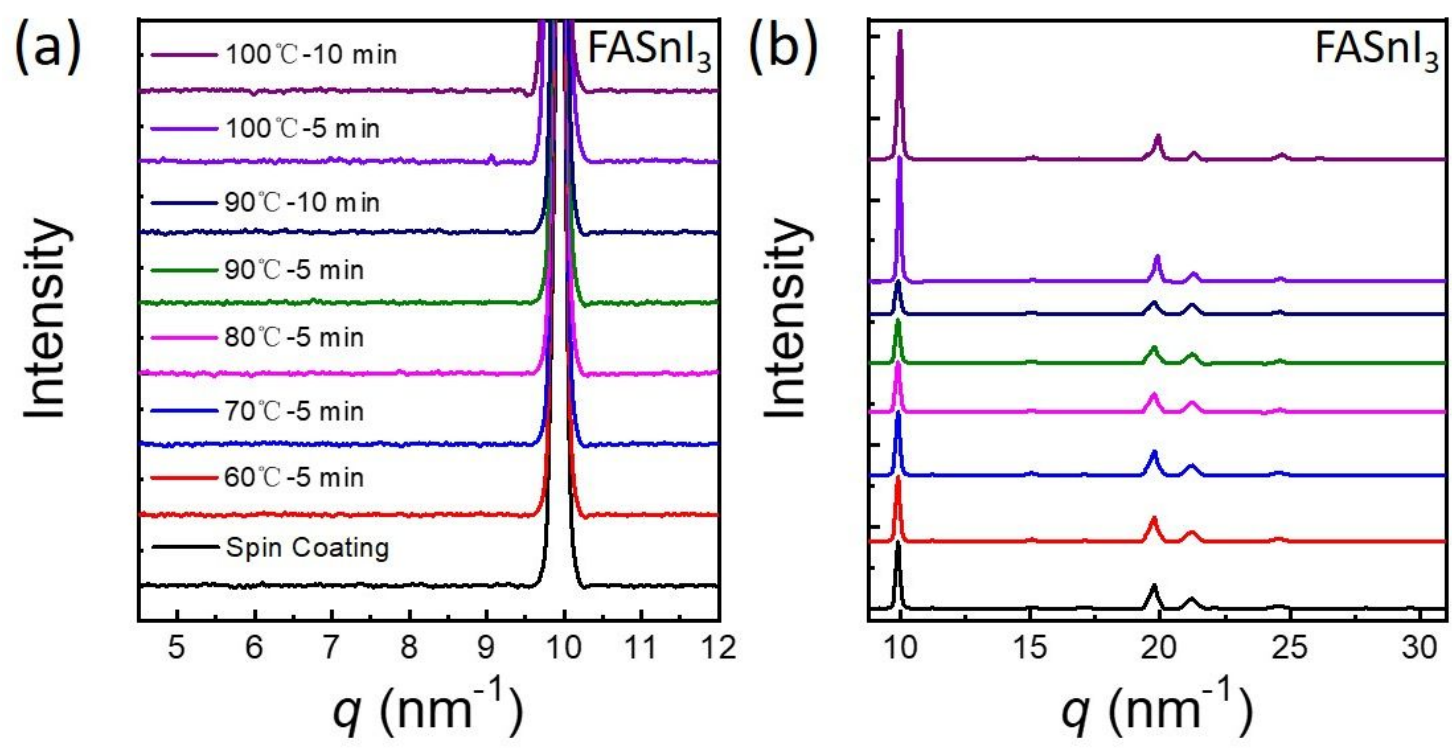

Figure S5. Azimuthally integrated intensity plots for the surface 2D GIXRD patterns along the direction of the outside surface (azimuth $90^{\circ}$ ) for $\mathrm{FASnI}_{3}$ films with different annealing temperatures. 

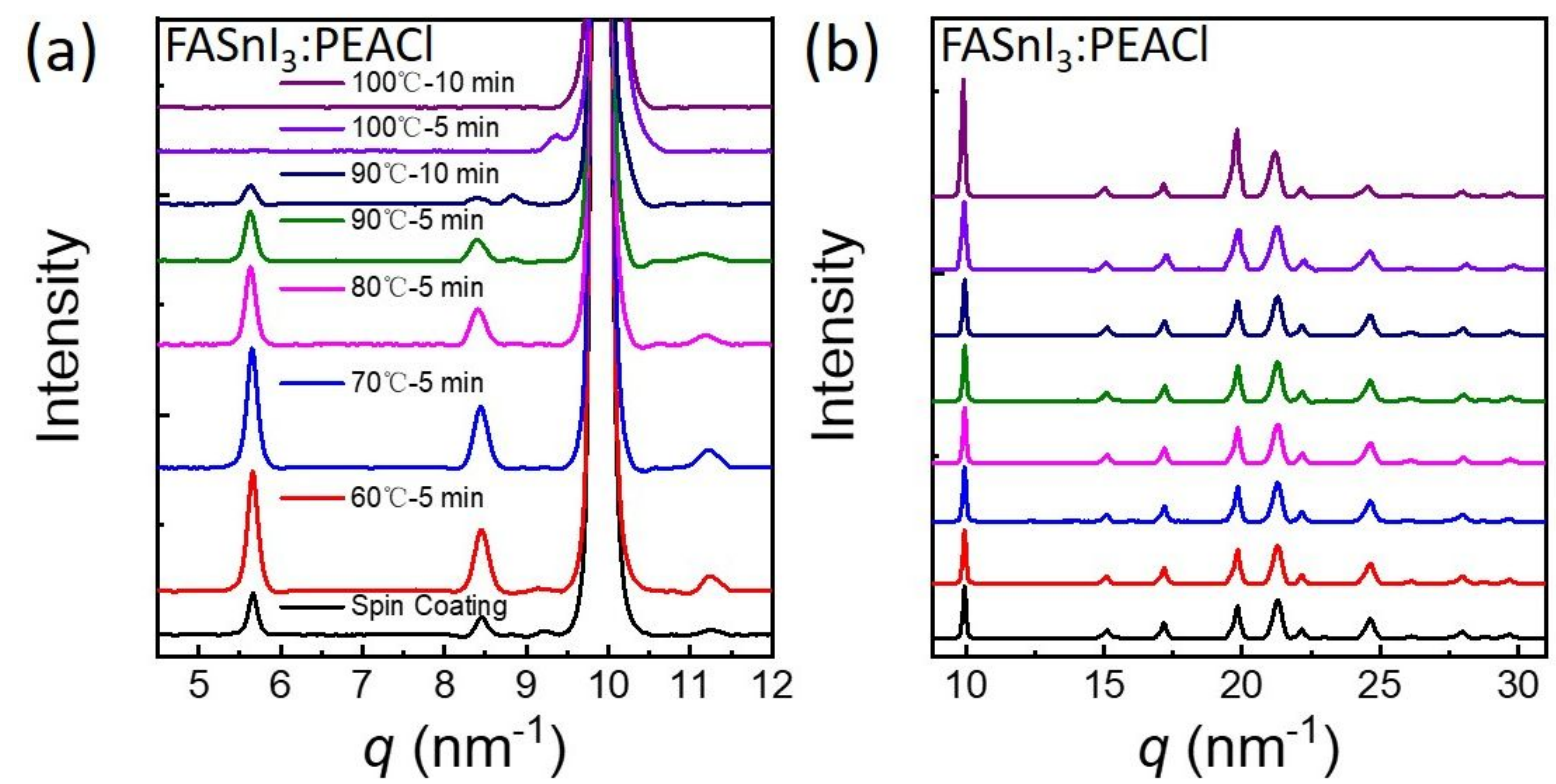

Figure S6. Azimuthally integrated intensity plots for the surface 2D GIXRD patterns along the direction of the outside surface (azimuth $90^{\circ}$ ) for $\mathrm{FASnI}_{3}: \mathrm{PEACl}$ films with different annealing temperatures. 


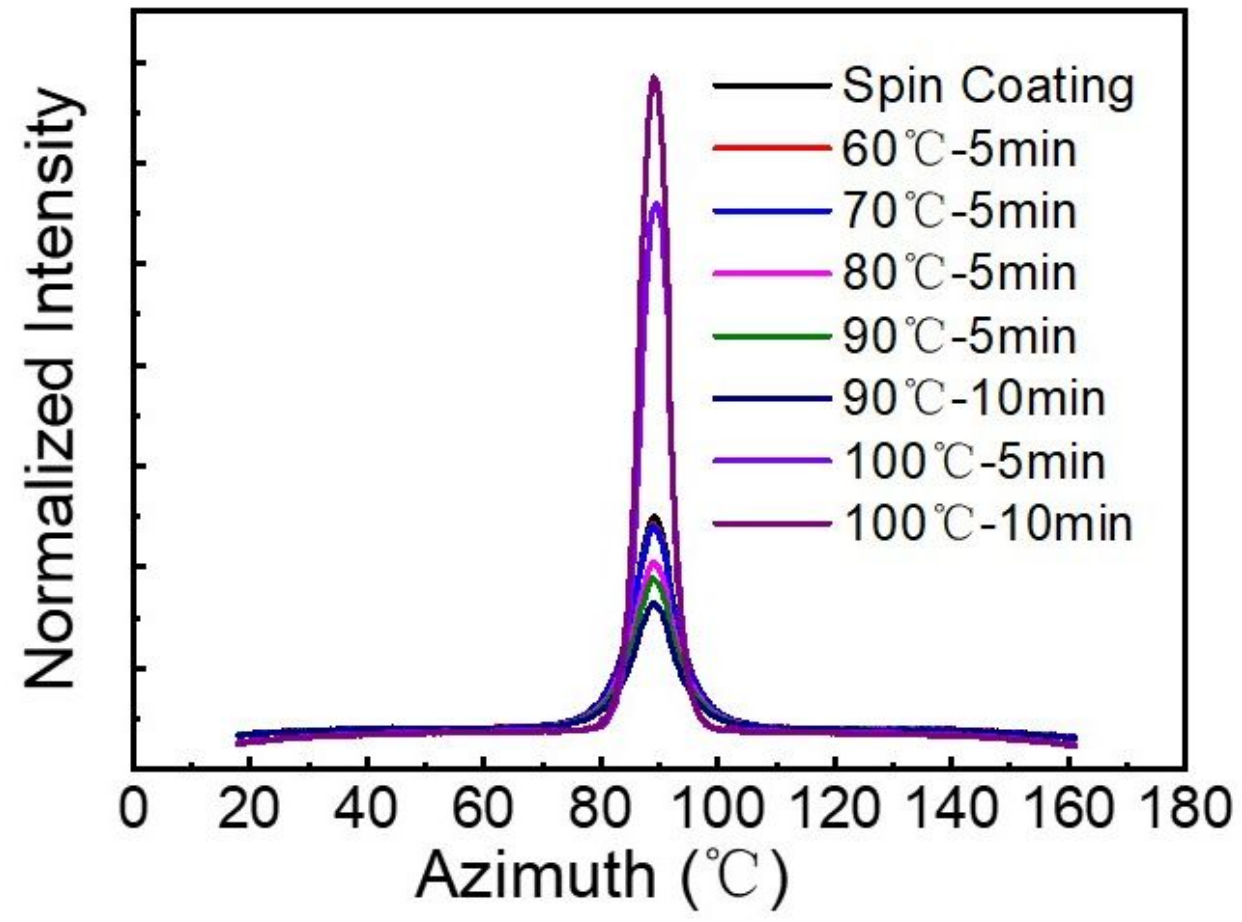

Figure S7. Radially integrated intensity plots along the ring at $q=10 \mathrm{~nm}^{-1}$, assigned to the (111) plane of $\mathrm{FASnI}_{3}: \mathrm{PEACl}$ with different annealing temperatures. 


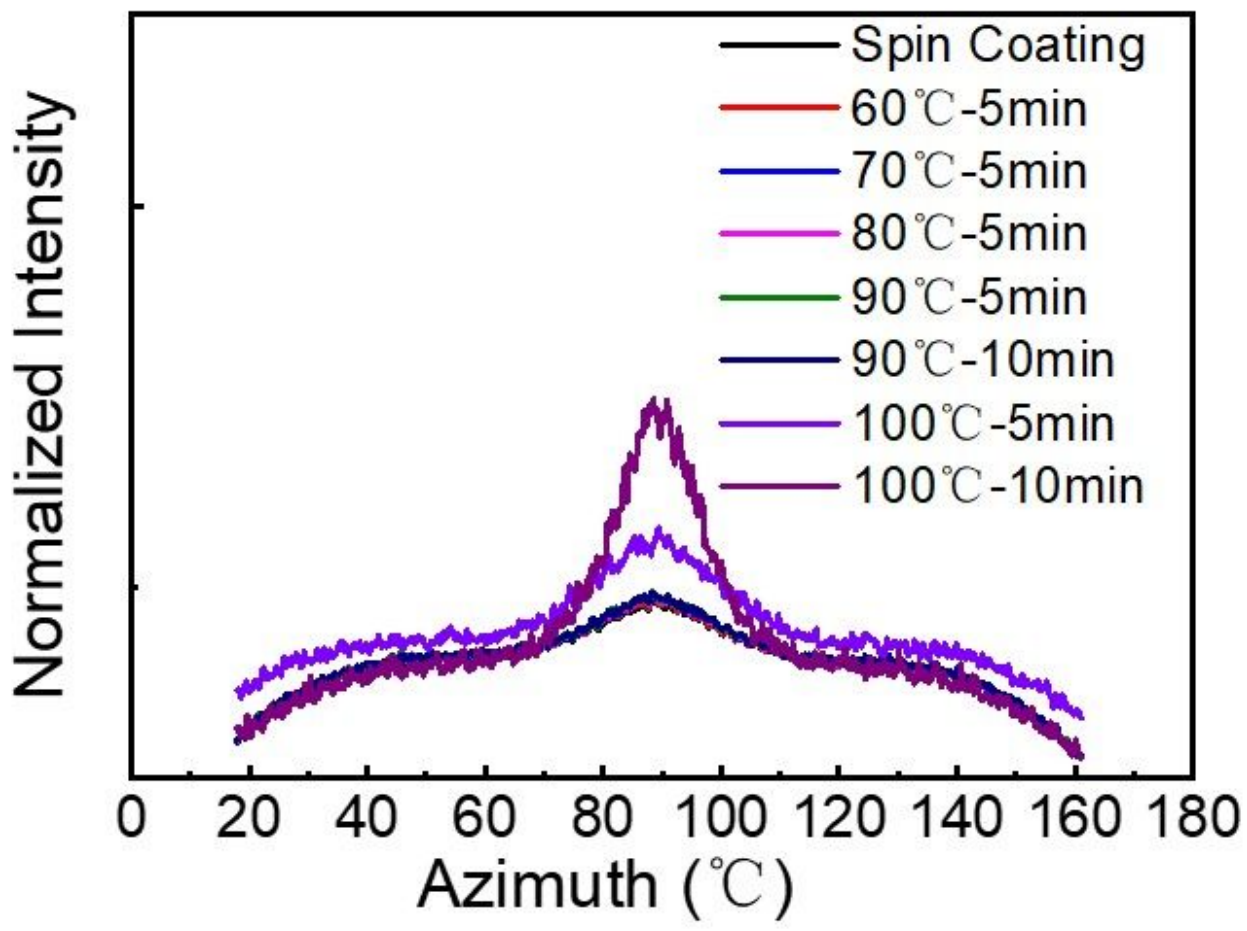

Figure S8. Radially integrated intensity plots along the ring at $q=10 \mathrm{~nm}^{-1}$, assigned to the (111) plane of $\mathrm{FASnI}_{3}$ with different annealing temperatures. 


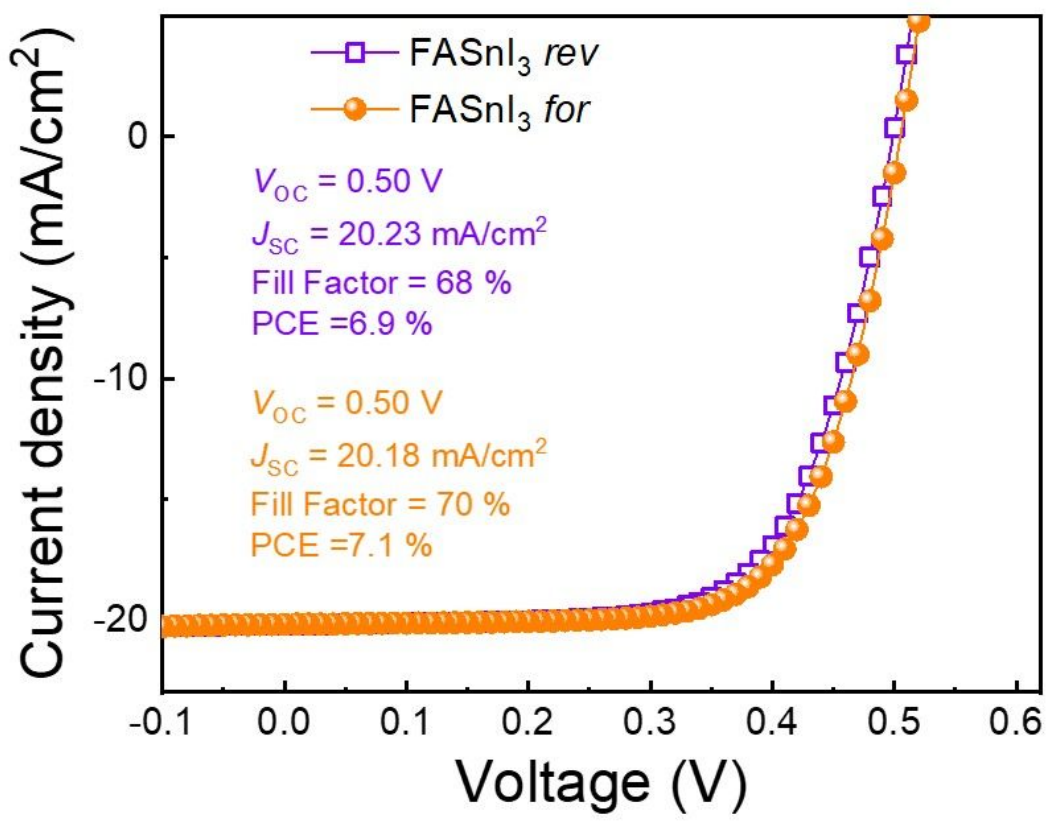

Figure S9. Forward and reverse sweep $J-V$ curves of the optimal device of $\mathrm{FASnI}_{3}: \mathrm{PEACl}$ PSCs with PEDOT:PSS hole transport layers. 

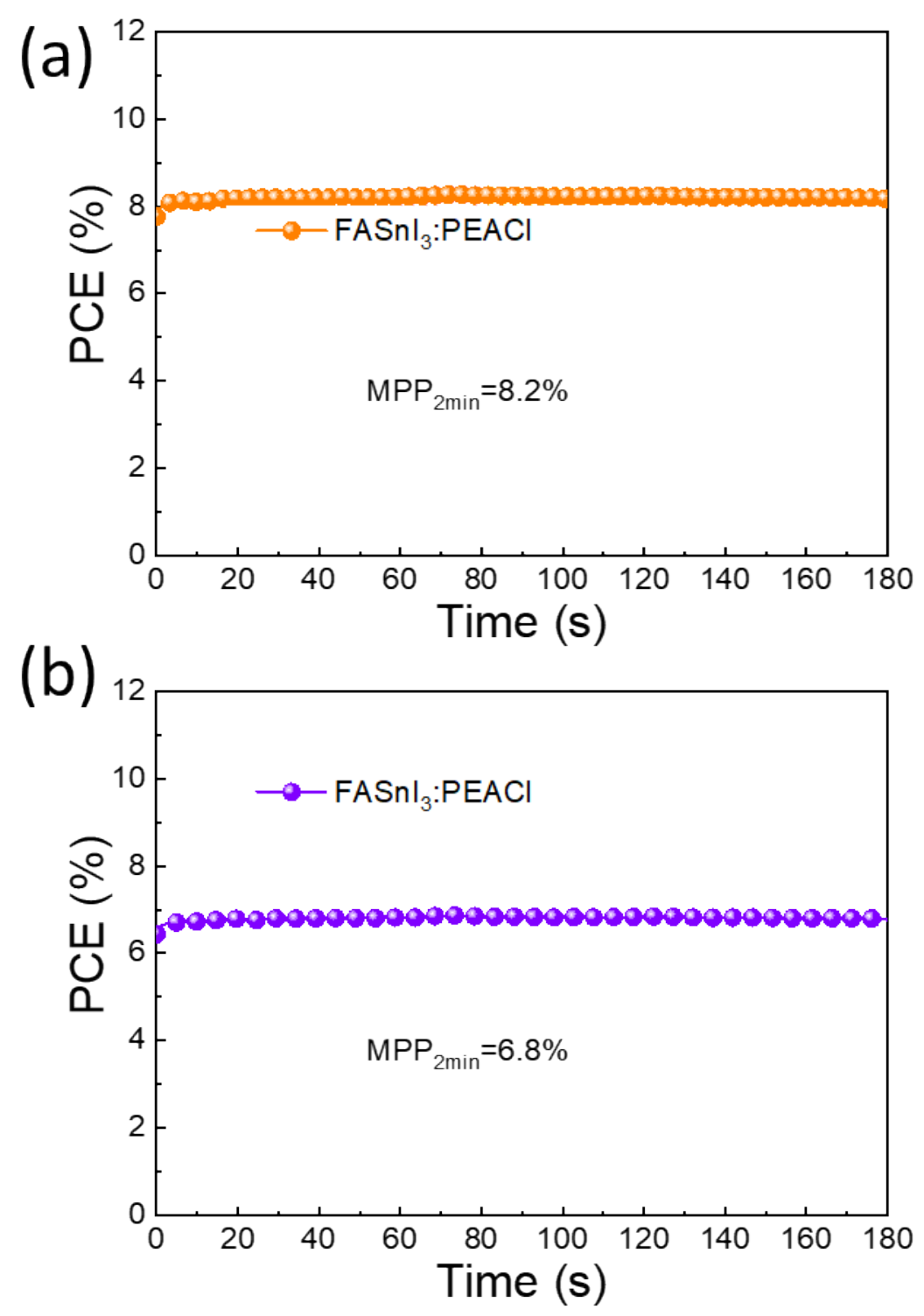

Figure S10. Steady-state efficiency of the optimized FASnI $3: \mathrm{PEACl}$ PSCs with (a) $\mathrm{NiO}_{\mathrm{x}}$ and (b) PEDOT:PSS hole transport layers at the maximum power point. 
Table S1. Performance parameters of $\mathrm{FASnI}_{3}$ perovskite solar cells without and with $\mathrm{PEACl}$ at different concentrations.

\begin{tabular}{|c|c|c|c|c|}
\hline Active layer & $\begin{array}{c}J_{\mathrm{sc}} \\
\left(\mathrm{mA} / \mathrm{cm}^{2}\right)\end{array}$ & $\begin{array}{l}V_{\mathrm{oc}} \\
(\mathrm{V})\end{array}$ & $\begin{array}{l}\mathrm{FF} \\
(\%)\end{array}$ & $\begin{array}{c}\text { PCE } \\
(\%)\end{array}$ \\
\hline $\mathrm{FASnI}_{3}$ for & 19.7 & 0.36 & 60.3 & 4.3 \\
\hline $\mathrm{FASnI}_{3}$ rev & 19.9 & 0.35 & 56.5 & 4.0 \\
\hline $\mathrm{FASnI}_{3}: \mathrm{PEACl} 4 \% \mathrm{~mol}$ for & 18.9 & 0.42 & 58.4 & 4.7 \\
\hline $\mathrm{FASnI}_{3}: \mathrm{PEACl} 4 \% \mathrm{~mol} \mathrm{rev}$ & 19.0 & 0.40 & 53.2 & 4.0 \\
\hline $\mathrm{FASnI}_{3}: \mathrm{PEACl} 8 \% \mathrm{~mol}$ for & 19.3 & 0.46 & 62.1 & 5.5 \\
\hline $\mathrm{FASnI}_{3}: \mathrm{PEACl} 8 \% \mathrm{~mol} \mathrm{rev}$ & 19.4 & 0.45 & 59.6 & 5.3 \\
\hline $\mathrm{FASnI}_{3}: \mathrm{PEACl} 12 \% \mathrm{~mol}$ for & 19.4 & 0.49 & 69.4 & 6.6 \\
\hline $\mathrm{FASnI}_{3}: \mathrm{PEACl} 12 \% \mathrm{~mol} \mathrm{rev}$ & 19.4 & 0.48 & 67.8 & 6.4 \\
\hline $\mathrm{FASnI}_{3}: \mathrm{PEACl} 16 \% \mathrm{~mol}$ for & 18.4 & 0.49 & 56.5 & 5.1 \\
\hline $\mathrm{FASnI}_{3}: \mathrm{PEACl} 16 \% \mathrm{~mol} \mathrm{rev}$ & 18.5 & 0.48 & 54.6 & 4.9 \\
\hline
\end{tabular}

Table S2. Performance parameters of $\mathrm{FASnI}_{3}: \mathrm{PEACl}$ perovskite solar cells with different annealing processes.

\begin{tabular}{|c|c|c|c|c|}
\hline Active layer & $\begin{array}{c}J_{\mathrm{sc}} \\
\left(\mathrm{mA} / \mathrm{cm}^{2}\right)\end{array}$ & $\begin{array}{l}V_{\mathrm{oc}} \\
(\mathrm{V})\end{array}$ & $\begin{array}{l}\mathrm{FF} \\
(\%)\end{array}$ & $\begin{array}{c}\text { PCE } \\
(\%)\end{array}$ \\
\hline $\mathrm{FASnI}_{3} 100^{\circ} \mathrm{C}$ for & 19.7 & 0.36 & 60.3 & 4.3 \\
\hline $\mathrm{FASnI}_{3} 100^{\circ} \mathrm{C} \mathrm{rev}$ & 19.9 & 0.35 & 56.5 & 4.0 \\
\hline $\mathrm{FASnI}_{3}: \mathrm{PEACl} 12 \% \mathrm{~mol} 70^{\circ} \mathrm{C}$ for & 17.5 & 0.47 & 56.5 & 4.7 \\
\hline $\mathrm{FASnI}_{3}: \mathrm{PEACl} 12 \% \mathrm{~mol} 70^{\circ} \mathrm{C} \mathrm{rev}$ & 17.5 & 0.46 & 51.2 & 4.1 \\
\hline $\mathrm{FASnI}_{3}: \mathrm{PEACl} 12 \% \mathrm{~mol} 80^{\circ} \mathrm{C}$ for & 17.9 & 0.48 & 58.2 & 5.0 \\
\hline $\mathrm{FASnI}_{3}: \mathrm{PEACl} 12 \% \mathrm{~mol} 80^{\circ} \mathrm{C} \mathrm{rev}$ & 17.9 & 0.48 & 56.9 & 4.9 \\
\hline $\mathrm{FASnI}_{3}: \mathrm{PEACl} 12 \% \mathrm{~mol} 90^{\circ} \mathrm{C}$ for & 19.7 & 0.49 & 67.2 & 6.5 \\
\hline $\mathrm{FASnI}_{3}: \mathrm{PEACl} 12 \% \mathrm{~mol} 90^{\circ} \mathrm{C} \mathrm{rev}$ & 19.7 & 0.48 & 65.0 & 6.2 \\
\hline $\mathrm{FASnI}_{3}: \mathrm{PEACl} 12 \% \mathrm{~mol} 100^{\circ} \mathrm{C}$ for & 19.4 & 0.49 & 69.4 & 6.6 \\
\hline $\mathrm{FASnI}_{3}: \mathrm{PEACl} 12 \% \mathrm{~mol} 100^{\circ} \mathrm{C} \mathrm{rev}$ & 19.4 & 0.48 & 67.8 & 6.4 \\
\hline
\end{tabular}




\section{REFERENCES}

(1) Kuai, L.; et al. Revealing crystallization dynamics and the compositional control mechanism of 2D perovskite film growth by in situ synchrotron-based gixrd. ACS Energy Lett. 2019, 5, 8-16.

(2) Jiang, F.; et al. Post-treatment-free solution-processed non-stoichiometric nio nanoparticles for efficient hole-transport layers of organic optoelectronic devices. Adv. Mater. 2015, 27, 2930. 